YANXI HAO, Research Assistant ${ }^{1}$

E-mail: haoyx0316@163.com

JING TENG, Ph.D. ${ }^{1}$

(Corresponding Author)

E-mail: tengjing@tongji.edu.cn

YINSONG WANG, Ph.D. ${ }^{2}$

E-mail: wangyinsong@siac-sh.com

XIAOGUANG YANG, Professor $^{1}$

E-mail: yangxg@tongji.edu.cn

${ }^{1}$ Key Laboratory of Road and Traffic Engineering of the

Ministry of EducationTongji University, 4800 Cao'an Road,

Shanghai, P. R. China

2 Shanghai International Automobile City (Group) Co., Ltd

No.888, South Moyu Road, Jiading District,

Shanghai, P.R.China
Traffic Management

Preliminary Communication

Submitted: Nov. 11, 2015

Accepted: July 14, 2016

\title{
INCREASING CAPACITY OF INTERSECTIONS WITH TRANSIT PRIORITY
}

\begin{abstract}
Dedicated bus lane ( $D B L$ ) and transit signal priority (TSP) are two effective and low-cost ways of improving the reliability of transits. However, these strategies reduce the capacity of general traffic. This paper presents an integrated optimization (IO) model to improve the performance of intersections with dedicated bus lanes. The 10 model integrated geometry layout, main-signal timing, pre-signal timing and transit priority. The optimization problem is formulated as a Mix-Integer-Non-Linear-Program (MINLP) that can be transformed into a Mix-Integer-Linear-Program (MILP) and then solved by the standard branch-and-bound technique. The applicability of the 10 model is tested through numerical experiment under different intersection layouts and traffic demands. A VISSIM micro simulation model was developed and used to evaluate the performance of the proposed 10 model. The test results indicate that the proposed model can increase the capacity and reduce the delay of general traffic when providing priority to buses.
\end{abstract}

\section{KEY WORDS}

urban roads; effective transit priority; dedicated bus lane; dynamic lane assignment;

\section{INTRODUCTION}

Provision of highly reliable transit services has been widely realized as an effective way to reduce traffic pressure on congested urban roads [1]. To improve the reliability of transits, transit signal priority (TSP) and dedicated bus lane (DBL) were proposed to optimize the allocation of space and time resources on the road. The DBLs provide dedicated lanes to avoid mixing buses with general traffic, and with TSP buses can pass the intersection with a requested priority signal phase. Due to their low-cost feature and effectiveness for mitigating traffic congestion, DBL and TSP have become pervasive throughout the major cities in the world. Especially in China, among the 70 major cities, more than $80 \%$ of them have DBLs and $40 \%$ have bus rapid transit (BRT) that are often provided with TSP.

Properly designed DBL and TSP can significantly improve transit reliability in terms of reducing delay and travel time fluctuation of buses caused by general traffic and signal control. However, most of the implementations failed to consider the capacity and delay of general traffic. The method used to provide a highly efficient TSP usually changed the signal control timing dramatically, which brought negative impacts on general traffic regarding the dimension of time. The DBL occupied the lanes for buses only, which disturbed the general traffic regarding the dimension of space. To overcome the drawbacks of transit priority, this paper focused on providing bus priority on dedicated bus lanes as well as maximizing the capacity of intersections. An integrated optimization (IO) method for lane assignment and traffic signal control has been developed in this paper based on the concept of using one or more of general traffic lanes as variable lanes for both left-turn traffic and through traffic. The optimization model is formulated as a Mix-Integer-Non-Linear-Program (MINLP), in which the lane assignment, main-signal timing, pre-signal timing and transit priority are integrated. The applicability and performance of the proposed 10 model is tested by numerical experiments and VISSIM simulation under different geometric layouts, bus priority demands and flow rates of general traffic.

The rest of this paper is organized as follows. The literature is reviewed on research of DBL, TSP, variable lanes and pre-signal control. The development of 10 method is introduced, including the concept of the IO scenario, the MINLP model and the solution 
method. The evaluation methods and test results of the proposed method are then presented. Conclusions and recommendations for the future work are given in the final section.

\section{LITERATURE REVIEW}

Smith Wilbur conducted the earliest bus pre-emption experiment, which gained significant reduction on buses travel time [1]. A large number of studies followed and focused on TSP strategies. Many of these studies focused on TSP timing strategies [2, 3], and the comparison and evaluation of the proposed strategies using either simulation or field data [4-9]. Conditional transit signal priority strategies were provided according to the bus schedule and provided only if the buses were behind the schedule [10-13], which would reduce the ineffective signal priority. Ma et al. [12] established a linear model to generate TSP strategies in case the priority was effective. Hao et al. [13] proposed a TSP method based on transit schedule, which provided priority between two successive bus stops, and calculated bus priority time from the last bus stop to the first one in order to keep the TSP effective. Besides TSP, DBL is also an effective measure in providing transit priority and improve transit reliability $[3,6,14]$. Combined with intermittent bus lane (IBL), DBL shows its benefit in transit priority as well as in reducing disturbance to general traffic flows $[15,16]$.

Variable lane is an effective measure addressing the problem of traffic flow changing at different time periods and the problem of congestion. Daganzo et al. [17] introduced the concept and implementation method of dynamic lane assignment for freeway congestion mitigation as part of the California PATH Program of the University of California. Lam et al. [18] developed a model integrating both the design of land-use and signal-phase control at isolated intersections and performed the optimization simultaneously to produce a better overall result. Zhao et al. [19] developed a two-step optimization model for dynamic lane assignment at isolated signalized intersections to solve the congestion problem. The arm-group-based model overcame the shortcoming of static lane marking and fully utilized the existing traffic facilities. Zhao et al. [20] also proposed an unconventional approach, which used the exit lanes for left-turn traffic dynamically, to increase the capacity of heavily congested intersections. Li et al. [21] developed a variable lane control strategy that attributed lanes and generated signal control method according to the detector data. The results of the simulation experiment showed that the strategy could increase capacity and reduce delay. The implementations of the variable lane assignment began in France in the 1960s, and two important trails are currently under way in the Paris region and the
Grenoble conurbation. The aim of the trails is to gain profit overall rather than locally [22].

Pre-signal as one of the means to provide TSP has been studied and implemented widely. The pre-signals have been used in Europe to provide transit priority for a long time [23]. Wu and Hornsell [24] proposed three categories of pre-signal based on a number of pre-signals that have been installed in the U.K., particularly in London. Buses in this study are subjected to the pre-signal as well as general traffic, which would provide partly priority to buses. Long [25] proved that the pre-signal control for buses in a real intersection performed better than passive bus priority, in terms of delay per person and vehicle. Xuan \& Daganzo et al. [26] proposed a method that reorganized all the lanes upstream of an intersection using a mid-block pre-signal. The result shows that if drivers behave properly, the increasing of the capacity could be significant. This method has proven to be efficient but with a flaw that it requires the interval length of two successive intersections. Wang [27] developed a lane-based optimization model of signal timings, which considered lane markings and signal timings at the same time to improve the operation of intersections.

In summary it can be concluded that the TSP and DBL strategies have been widely explored; the impacts of TSP and DBL on general traffic have been evaluated, but not considered in the optimization method in previous studies. There have been a significant number of studies focusing on using the variable lane and pre-signal strategies to improve the performance of intersection operation. The primary contribution of this paper is to provide bus signal priority on dedicated bus lanes as well as maximizing the capacity of intersections by integrating lane assignment and traffic signal control.

\section{THE CONCEPT AND GENERAL NOTATION}

\subsection{Basic concept of IO control}

Both TSP and DBL strategies cause reduction of the capacity for general traffic. The basic idea of the IO model is to use one or more general lanes as variable lanes, which is designed to respond to stochastic traffic demand and alleviate capacity reduction when transit priority is provided. As shown in Figure 1, pre-signals are set on the upstream of the variable lane to announce the right-of-way. The variable lane function is activated only when an approaching bus is requesting signal priority. Bus detector should be located no later than the exit lane of the upstream intersection, and the earlier the better. When bus priority is requested, the IO model will provide an optimized signal-timing plan, including pre-signal and main signal. 
The pre-signal plan includes the use plan of variable lane and the duration time for each movement. The strategy used to calculate the bus priority time was proposed in a previous work [13], which provide effective bus priority under the constraints of bus schedule adherence or headway stability at intersections between two successive bus stops.

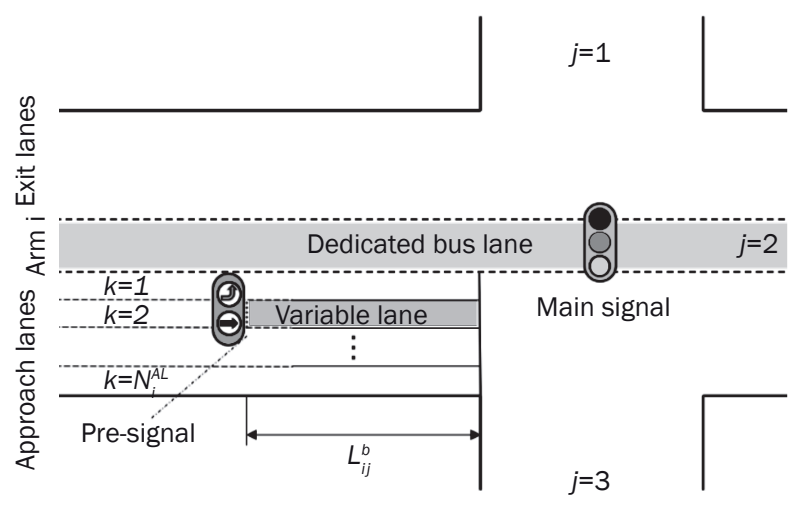

Figure 1 - Concept of integrated optimization control

Figure 2 shows the process of the 10 control method when bus priority is requested. The variable lanes may be used more than once in one cycle according to the optimized method. In a conventional intersection with four arms, the variable lanes can be used as both left-turn traffic lane and through traffic lane. Arrows are given as pre-signals for the announcement of the right-of-way. The green arrow for each movement will light up according to the corresponding phases in the main signal, and other movements receive a red arrow. Lane clearance is also considered when exchanging the right-of-way.

\subsection{Model assumptions}

In this paper, buses operate in a dedicated bus lane and only one transit priority request can be served in one cycle. The first-come-first-serve rule is applied for multiple priority requests situation. The speed of buses is considered as a constant. To decrease the impacts of pedestrians and bikes on buses, only the bus lane in the middle of the road is considered in this paper. With the correction factor of bikes and pedestrians considered, the model can be applied to both setting form of bus lanes, bus lanes in the middle of the road or on the side of the road.

The assumptions are suitable for DBL operation, bus rapid transit (BRT) system and tram with dedicated lanes. The model is still suitable under the mixed traffic situation with the bus travel time model amended. The amendment should consider the bus speed fluctuation and queue length caused by general traffic.

The notations used hereafter are summarized in Table 1.

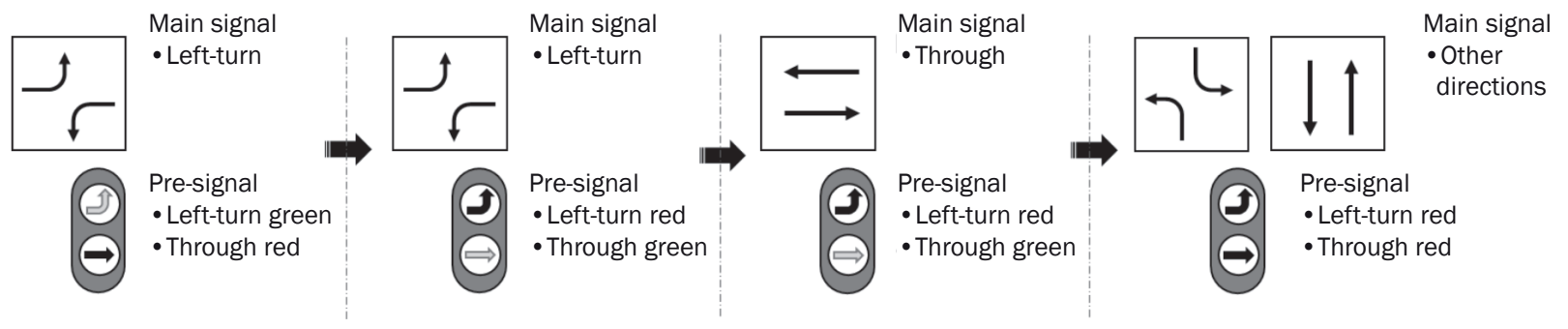

Figure 2 - Operating process of 10 model

Table 1 - Notations and variables

\begin{tabular}{||l|l||}
\hline Symbol & \multicolumn{1}{|c||}{ Definition } \\
\hline \hline$C_{\max }$ & Maximum of cycle length (s) \\
\hline$C_{\min }$ & Minimum of cycle length (s) \\
\hline$e^{b}$ & The request of bus priority \\
\hline$e$ & Extra effective green time that derives from the difference between actual and effective greens $(s)$ \\
\hline$g_{i j}$ & Minimum green duration for movement $j$ in arm $i$ \\
\hline$L_{i}^{c}$ & Length of variable lanes in arm $i$ \\
\hline$L_{i j}^{b}$ & Bus travel length from the location bus detected to the intersection for bus movement $j$ in arm $i$ \\
\hline$N_{j}^{E L}$ & Number of exit lanes in arm $j$ \\
\hline$P_{i}$ & Transit priority strategies $i$ \\
\hline
\end{tabular}


Y. Hao, J. Teng, Y. Wang, X. Yang: Increasing Capacity of Intersections with Transit Priority

\begin{tabular}{|c|c|}
\hline Symbol & Definition \\
\hline 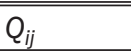 & Demand flow of movement $j$ in arm $i($ veh/h) \\
\hline$T_{i j}^{b}$ & Bus travel time to the intersection from the time bus detected for bus movement $j$ in arm $i$ \\
\hline$q_{i j k}$ & Assigned flow of movement $j$ on $k$ lane in arm $i(v e h / h)$ \\
\hline$q^{\prime} i j k$ & Assigned flow of movement $j$ on $k$ lane in arm $i$ when bus priority provided (veh/h) \\
\hline$s_{i k}$ & Saturation flow rate of lane $k$ in leg $i$ \\
\hline$v^{b}$ & Bus travel speed \\
\hline$y_{i c}$ & Flow ratio of variable lanes in leg $i$ \\
\hline$y_{i k}$ & Flow ratio of lane $k$ in leg $i$ \\
\hline$\delta_{i j k}$ & The permission of movement $j$ on $k$ lane in arm $i$ \\
\hline$\zeta^{p}$ & Reciprocal of pre-signal cycle length (1/s) \\
\hline$\theta_{i j}$ & Start of green for movement $j$ in arm $i$ \\
\hline $\overrightarrow{\theta^{\prime}}{ }_{m l}$ & Start of green for movement $j$ in arm $i$ when bus priority requested \\
\hline$\rho_{i j}$ & Bus detected time for bus movement $j$ in arm $i$ \\
\hline$\sigma_{i j}$ & The permission of movement $j$ on variable lanes in arm $i$ \\
\hline$\tau_{p q}^{b}$ & Bus arrival time at intersections for bus movement $p$ in arm $q$ \\
\hline$\omega_{i}^{c}$ & The ratio of clearance time of variable lanes in arm $i$ \\
\hline$\phi_{i j}$ & Duration of green for movement $j$ in arm $i$ \\
\hline$\phi_{i j}^{\prime}$ & Duration of green for movement $j$ in arm $i$ when bus priority requested \\
\hline$\Delta_{1}$ & Priority time 1 \\
\hline$\Delta_{2}$ & Priority time 2 \\
\hline$\Theta_{i c}$ & Start of green for variable lanes in arm $i$ \\
\hline$\Theta_{i k}$ & Start of green on $k$ lane in arm $i$ \\
\hline$\Phi_{\text {ic }}$ & Duration of green for variable lanes in arm $i$ \\
\hline$\Phi_{i k}$ & Duration of green on $k$ lane in arm $i$ \\
\hline$\Omega_{i j l m}$ & $\begin{array}{l}\text { Order of signal phase for a pair of mutually incompatible traffic movements }(i, j) \text { and }(I, m) \text {, if the start of green } \\
\text { of signal phase }(I, m) \text { follows that of signal phase }(i, j) \text {, and for the reverse order }\end{array}$ \\
\hline Y & Flow ratio reduction coefficient of variable lanes \\
\hline$\varepsilon$ & Allowing error of signal control timing \\
\hline$\zeta$ & Reciprocal of main-signal cycle length (1/s) \\
\hline$\eta$ & Priority time for buses that can be optimized \\
\hline$\mu$ & Common flow multiplier \\
\hline$\psi \mathrm{s}$ & Set of incompatible signal groups \\
\hline
\end{tabular}

\section{METHODOLOGY}

\subsection{Objective function}

Three criteria are usually employed for the optimization of signal settings: capacity maximization, cycle length minimization and delay minimization. The purpose of this paper is to maximize the capacity of intersections during provision of TSP. Therefore, the capacity maximization is chosen as the optimization objective. Adopting the commonly used assumption that the turn proportions would remain constant [27], maximizing the reserve capacity is equivalent to maximizing the common flow multiplier, $\mu$.

Maximize $\mu$

\subsection{Reserved capacity constraint}

The traffic demand is allocated to all the lanes in every arm. The maximum traffic volume that still allowed the intersection to operate reasonably well is defined as the traffic demand $Q$ multiplied by the flow multiplier $\mu$. The sum of the flow assigned to each lane equals the maximum traffic volume.

$$
\mu Q_{i j}=\sum_{k=1}^{N_{i}^{A L}} q_{i j k}, \forall i=1,2, \ldots, N_{A}, j=1,2, \ldots, N_{A}-1
$$

\subsection{Lane assignment constraints}

\section{1) Movement permitted on one lane}

Each traffic lane can permit only one movement to avoid conflict with buses on the dedicated bus lane, which can be specified as: 
$\sum_{j=1}^{N_{A-1}} \delta_{i j k}=1, \quad \forall i=1,2, \ldots, N_{A}, \quad k=1,2, \ldots, N_{i}^{A L}$

2) Prohibited movement

Only the movement permitted on a lane can be assigned to it, which can be specified as:

$$
\begin{aligned}
& M \delta_{i j k} \geq q_{i j k} \geq 0, \forall i=1,2, \ldots, N_{A}, j=1,2, \ldots, N_{A}-1, \\
& k=1,2, \ldots, N_{i}^{A L}
\end{aligned}
$$

where $M$ is an arbitrary large positive constant. If $\delta_{i j k}=0$, the movement is prohibited, and the assigned flow should be 0 . Otherwise, if $\delta_{i j k}=1$, the assigned flow can be any positive value as long as it satisfies other constraints.

\section{3) Permitted movements across adjacent lanes}

For safety reasons, any two adjacent lanes $k$ and $k+1$, from arm $i$, if the traffic movement $i \rightarrow j$ is permitted on the lane $k+1$; the movement $i \rightarrow j+1$ should be prohibited on lane $k$, which is shown as follows:

$$
\begin{aligned}
& 1-\delta_{i j k+1} \geq \delta_{i m k}, \forall i=1,2, \ldots, N_{A}, \quad j=1, \ldots, N_{A}-1, \\
& k=1, \ldots, N_{i}^{A L}, \quad m=j+1, \ldots, N_{A}
\end{aligned}
$$

4) Maximum permitted movements at the exit lanes

The number of exit lanes should be no less than the maximum number of entrance lanes that are assigned the flow to the exit lanes, which can be specified as the following equation:

$$
\begin{gathered}
N_{j}^{E L}=\max \left(\sum_{k=1}^{N_{i}^{A L}} \delta_{i j k}, \forall i=1,2, \ldots, N_{A},\right. \\
\left.j=1,2, \ldots, N_{A}-1, i \neq j\right)
\end{gathered}
$$

\subsection{Signal timing constraints}

\section{1) Intersection main-signal timing}

Cycle length: Let the maximum and minimum cycle lengths of the intersection be $C_{\max }$ and $C_{\min }$, respectively. Instead of defining the cycle length directly as a variable, its reciprocal $\zeta$, is used as the variable to preserve the linearity in the mathematical formulation.

$\frac{1}{C_{\min }} \geq \zeta \geq \frac{1}{C_{\max }}$

Start of green: since the signal timing at the intersection is cyclic in nature, the starts of green can be picked arbitrarily along the time axis as long as they satisfy other relevant constraints. However, for linearity reason, all of the starts of the green variables are confined to the range of $[0,1]$.

$1 \geq \theta_{i j \geq 0}, \forall i=1,2, \ldots, N_{A}, j=1,2, \ldots, N_{A}-1$

The start of green for the pair of movements from arm $i$ to $\operatorname{arm} j$ and from arm $j$ to arm $i$ should be equal when they are in the same direction with the bus operation $\left(i j^{b}\right)$. $\theta_{i j}=\theta_{j i}, \quad \forall i j=i j^{b}$

$$
\theta_{i j} \neq \theta_{i m}, \forall i j=i j^{b}, m=1, \ldots, N_{A}-1, m \neq j
$$

Duration of green: the duration of green is subjected to the minimum green time $g_{i j}$, to satisfy the safety and comfortable requirement and can be set as:

$1 \geq \phi_{i j} \geq g_{i j} \zeta, \forall i=1,2, \ldots, N_{A}, j=1,2, \ldots, N_{A}-1$

The duration of green for the pair of movement from arm $i$ to $\operatorname{arm} j$ and from $\operatorname{arm} j$ to $\operatorname{arm} i$ should be equal when they are in the same direction with the bus operation $\left(i j^{b}\right)$.

$\phi_{i j}=\phi_{j i}, \quad \forall i j=i j^{b}$

Sequence of signal phase: designate the set of incompatible signal groups as $\psi_{s}$. For any two conflict signal phases, $(I m)$ and $(i j)$ in $\psi_{s}$, the order of the signal displays is governed by a successor function [28], $\Omega_{i j / m}$; where $\Omega_{i j / m}=0$ represents the start of green of signal group $(I m)$ following that of the signal group (ij), and $\Omega_{i j / m}=1$ stands for the opposite. The following constraints can be set for the successor functions:

$\Omega_{i j l m}+\Omega_{l m i j}=1, \quad \forall(i j),(m l) \in \psi_{s}$

Lane signal settings: the signal timing of the movement in a lane should be the same with the intersection signal timings set for the movement, which can be specified as:

$M\left(1-\delta_{i j k}\right) \geq \Theta_{i k}-\theta_{i j} \geq-M\left(1-\delta_{i j k}\right)$

$M\left(1-\delta_{i j k}\right) \geq \Phi_{i k}-\phi_{i j} \geq-M\left(1-\delta_{i j k}\right)$

2) Pre-signal timing

The pre-signals are employed only when bus priority is requested, which can be described as $e^{b}=1$.

Cycle length: For coordination purpose, the cycle length of the pre-signal should be the same as the main signal.

$\zeta^{p}=\zeta$

Clearance time of variable lane: The clearance time is needed to empty the variable lane for different movements. For convenience, the ratio of the clearance time in a cycle is used as the constraint.

$\omega_{i}^{c}=\frac{\frac{L_{i}^{c}}{v^{g}}}{C}$

Start of green: The start of green of the movement $j$ on variable lane in arm $i$ can be calculated as follows, as long as they satisfy other relevant constraints.

$$
\begin{aligned}
& M\left(1-\delta_{i j k}\right)\left(1-\sigma_{i j}\right) e^{b} \geq \Theta_{i j}^{c}-\theta_{i j}-\omega_{i}^{c} \geq \\
& \geq-M\left(1-\delta_{i j k}\right)\left(1-\sigma_{i j}\right) e^{b}
\end{aligned}
$$

Duration of green: To keep the operation order of intersections, the green duration of movement $j$ on the 
variable lane in arm $i$ should not be larger than the main-signal.

$$
\begin{aligned}
& M\left(1-\delta_{i j k}\right)\left(1-\sigma_{i j}\right) e^{b} \geq \Phi_{i j}^{c}-\left(\phi_{i j}-\varepsilon\right) \geq \\
& \geq-M\left(1-\delta_{i j k}\right)\left(1-\sigma_{i j}\right) e^{b}
\end{aligned}
$$

\subsection{Flow ratio constraints}

\section{1) Flow ratio for normal lanes}

The flow ratio of a lane is the ratio of the flow rate to the saturation flow rate, which can be expressed as:

$y_{i k}=\frac{q_{i k}}{s_{i k}}, \quad \forall i=1,2, \ldots, N_{A}, k=1, \ldots, N_{i}^{A L}$

\section{2) Flow ratio for variable lanes}

Since frequent changing of the right-of-way on variable lanes may cause capacity reduction, the flow saturation of the variable lane should be reduced by coefficient $\gamma$.

$$
y_{i c}=\frac{q_{i k}}{s_{i k}} \cdot \gamma, \quad \forall i=1,2, \ldots, N_{A}, k=1, \ldots, N_{i}^{A L}
$$

3) Maximum acceptable degree of saturation

The maximum acceptable degree of saturation that ensures the intersection to perform well is defined as $Y_{\text {max }}$. The degree of saturation of each traffic lane should not be greater than the maximum acceptable degree of saturation.

$$
Y_{\max }=\frac{Y_{i k}}{\Phi_{i k}+e \zeta^{\prime}}, \forall i=1,2, \ldots, N_{A}, k=1, \ldots, N_{i}^{A L}
$$

\subsection{Transit signal priority constraints}

\section{1) Location of variable lanes}

When the approaching bus is requesting priority and the function of variable lane is activated, the location of the variable lane is decided as follows:

$$
\begin{aligned}
& \delta_{i j v}=\delta_{i j k}, \forall \delta_{i(j-1)(k-1)}>\delta_{i j k}=\delta_{i j(k+1)}, \\
& \forall i=1,2, \ldots, N_{A}, k=1,2, \ldots, N_{i}^{A L}
\end{aligned}
$$

The first lane with the same direction to bus lane is set to be the variable lane.

2) Bus travel time

A bus detector should be set at the exit bus lane of the upstream intersection. The travel time of the bus from where it is detected to the intersection can be calculated as:

$T_{i j}^{b}=\frac{L_{i j}^{b}}{V^{b}}, \quad \forall i=1,2, \ldots, N_{A}, j=1,2, \ldots, N_{A}-1$

\section{3) Available priority strategies}

Two types of transit signal priority strategies are employed in this paper, which are green extension and red truncation. The priority strategies can be specified as follows:

$P_{i}=\left\{\begin{array}{l}1, \text { Green extension } \\ 0, \text { No strategy } \\ -1, \text { Red truncation }\end{array}\right.$

\section{4) Priority strategies}

The priority time of a bus in a certain intersection is generated by the optimization model proposed in the previous work [13]. The optimization model provided effective bus priority at intersections between two successive bus stops according to the bus schedule.

To avoid severe changing of signals in intersections, coefficient $\eta$ is used to keep the available priority time in a relatively small range. For example, as shown in Figure $3, T_{1}^{d}, T_{2}^{d}, T_{3}^{d}$ are the bus detected time, $\eta$ is the available priority time. Suppose the buses are detected at $T_{1}^{d}$, no strategy will be taken, while if they are detected at $T_{2}^{d}$ or $T_{3}^{d}$, the strategies will be calculated as Equation 25. Solutions 1-4 in Figure 3 show the probable solutions of providing bus priority.

The priority time of a bus is decided by their detected time, travel time to the intersection and the available priority time, which can be specified as follows:

$\left\{\begin{array}{lll}\theta_{m l} \leq \tau_{p q}^{b} \leq \theta_{m l}+\phi_{m l}, & P_{i}=0 & \\ \theta_{m l}+\phi_{m l} \leq \tau_{p q}^{b} \leq \eta, & P_{i}=1, & \phi_{m l}^{\prime}=\phi_{m l}+\Delta_{1} \\ \eta \leq \tau_{p q}^{b} \leq \theta_{m l}+\zeta, & P_{i}=-1, & \theta_{m l}^{\prime}=\theta_{m l}-\Delta_{2}\end{array}\right.$

$\tau_{p q}^{b}=\rho_{i j}+\frac{T_{m l}^{b}}{C}$

$\Delta_{1}=\rho_{i j}+\frac{T_{m l}^{b}}{C}-\theta_{m l}-\phi_{m l}$

$\Delta_{2}=\theta_{m l}+\zeta-\rho_{i j}-\frac{T_{m l}^{b}}{C}$

5) Pre-signal signal timing

The pre-signal timing should be recalculated using the following equation after bus priority has been provided:

$$
\begin{aligned}
& \Theta_{i c}=\theta_{m l}^{\prime}-\omega_{c}, \quad P_{i} \neq 0 \\
& \Phi_{i c}=\phi_{m l}^{\prime}-\omega_{c}-\varepsilon, \quad P_{i} \neq 0
\end{aligned}
$$

6) Main signal timing

In response to the changing of traffic volume, the traffic flow and signal timing should be redistributed. To satisfy the signal timing and traffic flow requirement, the following constraints can be set: 


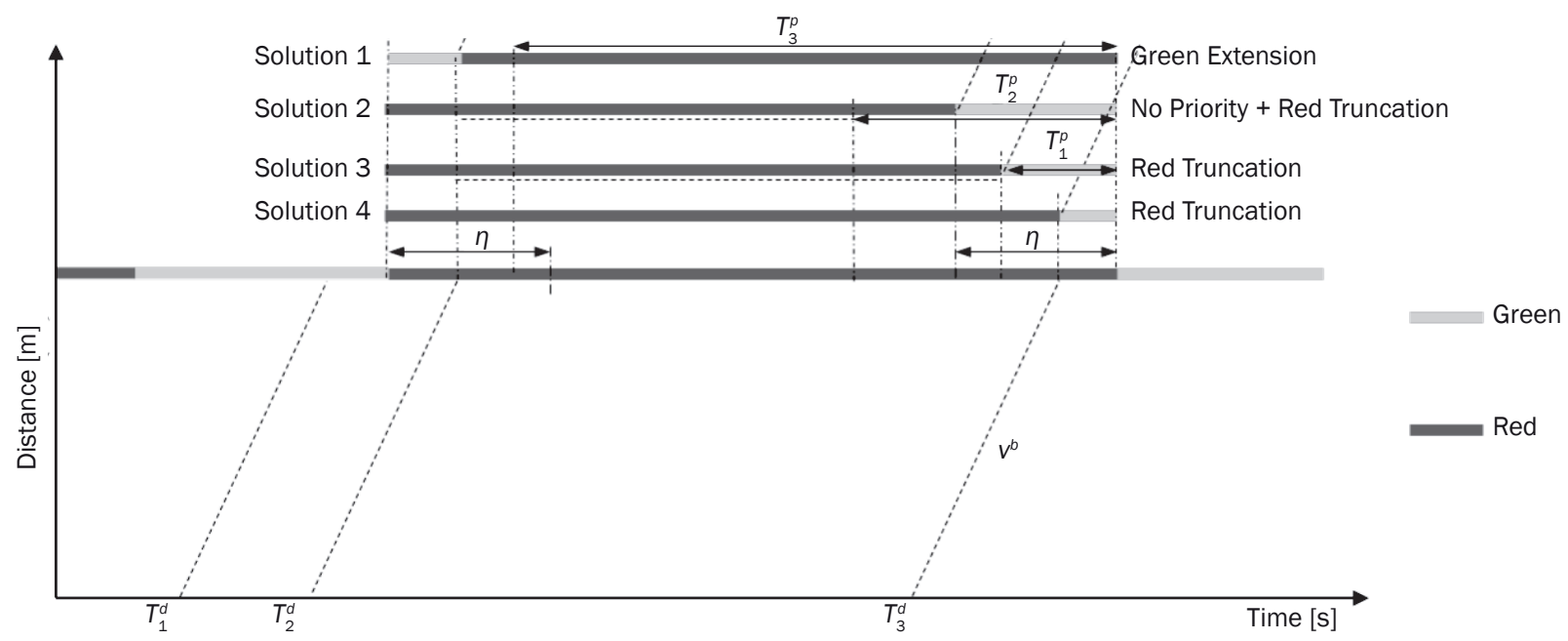

Figure 3 - Schematic plot of signal priority solutions

$\theta_{i j}^{\prime}=\rho_{m l}+\phi_{p q}^{\prime}$

$\phi_{i j}^{\prime}=\frac{\left(\frac{1}{\zeta}-\frac{1}{\zeta^{\prime}}\right)}{C} \phi_{i j}$

$q_{i j k}^{\prime}=q_{i j k}\left(2-\frac{\phi_{i j}^{\prime}}{\phi_{i j}}\right)$

\subsection{Solution method}

There are two main parts of the integrated optimization (IO) model: the signal timing part, including both main-signal and pre-signal, and the transit priority part. The 10 model is a Mix-Integer-Non-Linear-Program (MINLP) with the objective function as shown in Equation 1 and subjects to Equations 2-33. It is hard to obtain a global solution for this kind of problems. However, after examining the constraints, the variables that make the whole 10 model non-linear have been found, including the length of the variable lane $L_{i}^{c}$, and the length from the bus detected location to the intersection $L_{i j}^{b}$, which is often the length of the link between two successive intersections. For implementation, the link length $L_{i j}^{b}$ is often predetermined, and the length of the variable lane can be ranged in a set, which can be tried and put into the model by the brute force solution. The simplified method made the 10 model turn to a Mix-Integer-Linear-Program (MILP) which can be solved by the standard branch-and-bound technique.

The signal timings without bus priority are calculated by Equations 2-22. When bus priority is required, the signal timings and bus priority strategy should be recalculated by Equations 23-34. The bus priority strategy can be calculated as shown in Figure 4.

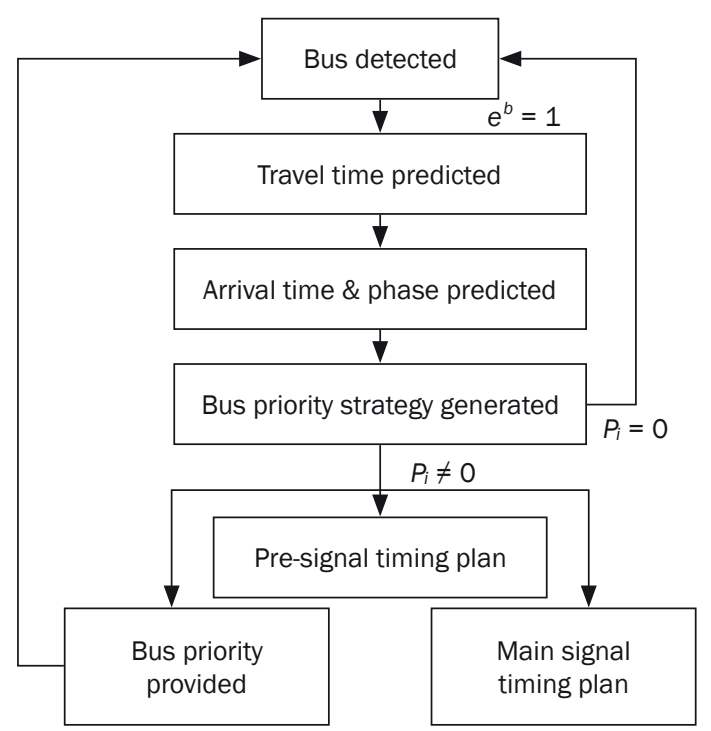

Figure 4 - Bus priority process

\section{APPLICABILITY ANALYSIS AND SIMULATION EVALUATION}

\subsection{Numerical analysis}

This section presents the evaluation of the performance of the proposed model by numerical analysis.

Consider a four-arm intersection with dedicated bus lane, as shown in Figure 5. Three cases with different numbers of through and left-turn lanes are studied as shown in Figure 5.

Common data input in all of the three cases is shown in Table 2. A waving area exists in the upstream of the variable lane. To maximize the adverse impact of the waving area and test the performance of the proposed model under a worse situation, the length of the variable lane is set at 50 meters and shorter than the queue length of that arm. 


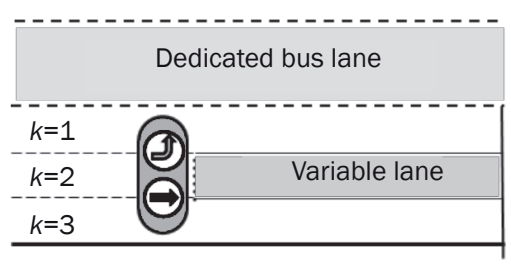

Case 1: $L(1)-V(1)-T(1)$

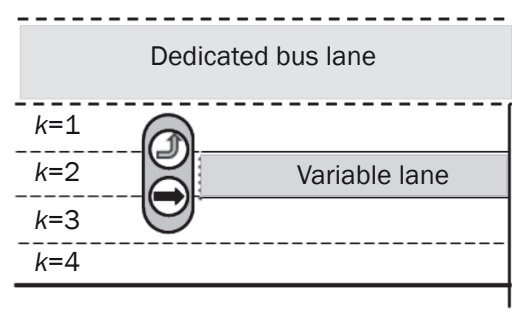

Case 2: $L(1)-V(1)-T(2)$

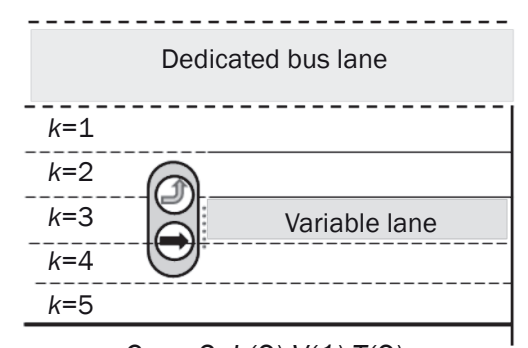

Case 3: $L(2)-V(1)-T(2)$

Figure 5 - Numerical analysis: variable lane layouts (L:left-turn; V:variable; T:through)

Table 2 - Common data input

\begin{tabular}{||l|l||}
\hline \multicolumn{1}{|c|}{ Variables Input } & \multicolumn{1}{c||}{ Value } \\
\hline \hline Number of arms & $N_{A}=4$ \\
\hline Original turning ratio & $\begin{array}{l}\text { Left-turn: } \\
\text { Through=1:2 }\end{array}$ \\
\hline $\begin{array}{l}\text { Maximum acceptable degree of } \\
\text { saturation }\end{array}$ & $Y_{\max }=90 \%$ \\
\hline Saturation flow rate & $\begin{array}{l}y_{i k}=y_{i c}=1,800 \\
(\mathrm{veh} / \mathrm{h})\end{array}$ \\
\hline Minimum duration of green & $g_{i j}=6(\mathrm{~s})$ \\
\hline $\begin{array}{l}\text { Extra effective green time that derives } \\
\text { from the difference between actual } \\
\text { and effective greens }\end{array}$ & $\mathrm{e}=1(\mathrm{~s})$ \\
\hline Speed of bus & $v^{b}=40(\mathrm{~km} / \mathrm{h})$ \\
\hline Speed of general traffic & $v^{g}=60(\mathrm{~km} / \mathrm{h})$ \\
\hline
\end{tabular}

The flow volume inputs are presented in Table 3. To identify the applicability of the 10 model, the conventional priority method and the 10 method have been compared. Variable lane strategy has been only employed in the 10 method, and bus priority has been employed in both $\mathrm{IO}$ and conventional priority method. The maximum cycle length is set to be 120 s.

1) Evaluation under different geometry layouts

Represented in Figure 6 is the capacity comparison between the 10 model and the conventional priority method under different geometry layouts in the three cases. The priority time for buses is described as the proportion to a cycle time which is in the range of [5\%,30\%] and the performance of the 10 model is tested along the priority proportion axis. The capacity ratio axis is the ratio of the 10 model to the conventional model. The conventional model differs from the 10 model in the geometry layout part, which means that no variable lanes are considered in the conventional model while the 10 model considered all factors described in Section 2.

As illustrated in Figure 6, the 10 strategy shows its effectiveness in increasing the capacity of intersections, especially in the intersections with high volume and several traffic lanes. The 10 method can increase the capacity of the intersection when bus priority is provided and performs better than the conventional priority method when the bus priority time is longer. The dissatisfactory performance of 10 model shown in Figure $6 c$ is analyzed to be caused by the reduction coefficient multiplied with the flow ratio of variable lanes.

Table 3 - Traffic demand under conventional situation

\begin{tabular}{|c|c|c|c|c|}
\hline $\begin{array}{l}\text { Traffic demand } \\
\text { [pcu/h] }\end{array}$ & \multicolumn{4}{|c|}{ To arm [number of lanes] } \\
\hline a) Case 1: left-turn (1) - variable (1) - through (1) \\
\hline $\begin{array}{l}\text { From arm } \\
\text { [number of lanes] }\end{array}$ & $1[3]$ & $2[2]$ & $3[3]$ & $4[2]$ \\
\hline \hline $1[3]$ & - & 250 & 500 & 200 \\
\hline $2[3]$ & 200 & - & 400 & 150 \\
\hline $3[3]$ & 400 & 200 & - & 200 \\
\hline $4[3]$ & 200 & 400 & 250 & - \\
\hline
\end{tabular}

b) Case 2: left-turn (1) - variable (1) - through (2

\begin{tabular}{|c|c|c|c|c|}
\hline $\begin{array}{l}\text { From arm } \\
\text { [number of lanes] }\end{array}$ & $1[4]$ & $2[2]$ & $3[4]$ & $4[2]$ \\
\hline \hline $1[4]$ & - & 350 & 700 & 200 \\
\hline $2[3]$ & 200 & - & 400 & 150 \\
\hline $3[4]$ & 700 & 200 & - & 350 \\
\hline $4[3]$ & 150 & 150 & 400 & - \\
\hline
\end{tabular}

c) Case 3: left-turn (2) - variable (1) - through (2)

\begin{tabular}{|c|c|c|c|c|}
\hline $\begin{array}{l}\text { From arm } \\
\text { [number of lanes] }\end{array}$ & $1[4]$ & $2[2]$ & $3[4]$ & $4[2]$ \\
\hline \hline $1[5]$ & - & 450 & 900 & 200 \\
\hline $2[3]$ & 400 & - & 200 & 150 \\
\hline $3[5]$ & 900 & 200 & - & 450 \\
\hline $4[3]$ & 150 & 150 & 400 & - \\
\hline
\end{tabular}

2) Evaluation under different flow ratios

Under the same geometry layout in Case 1, changing the ratio of the left-turn to through traffic and keeping the demand flow and cycle length the same are the basic concepts in this evaluation section.

The flow ratio of left-turn to through traffic differs from 1:1 to 1:3 as shown in Figure 7. As the left-turn demand increases, the capacity of the intersection firstly increases and then decreases. This phenomenon can be explained as follows: 


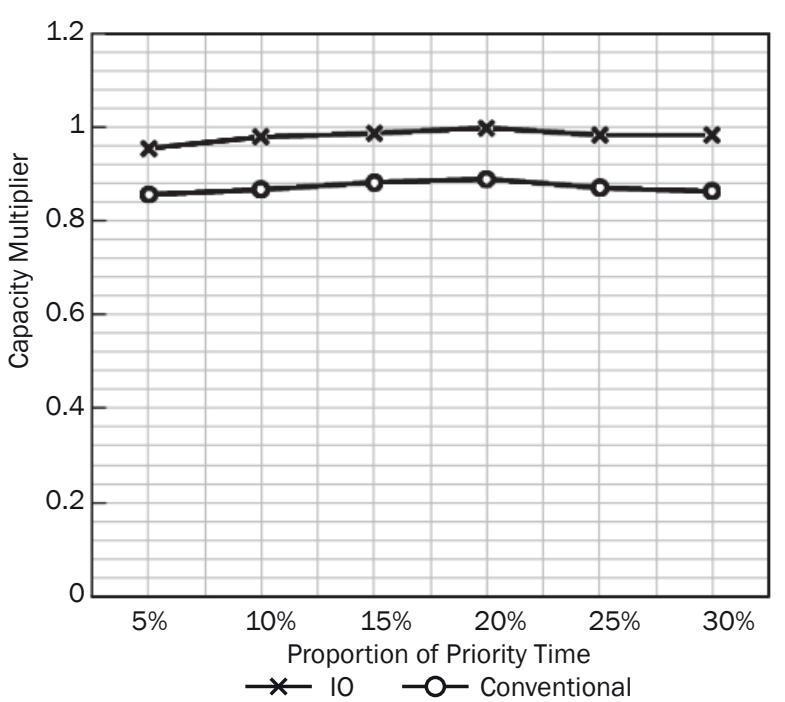

a) Case 1: $L(1)-V(1)-T(1)$
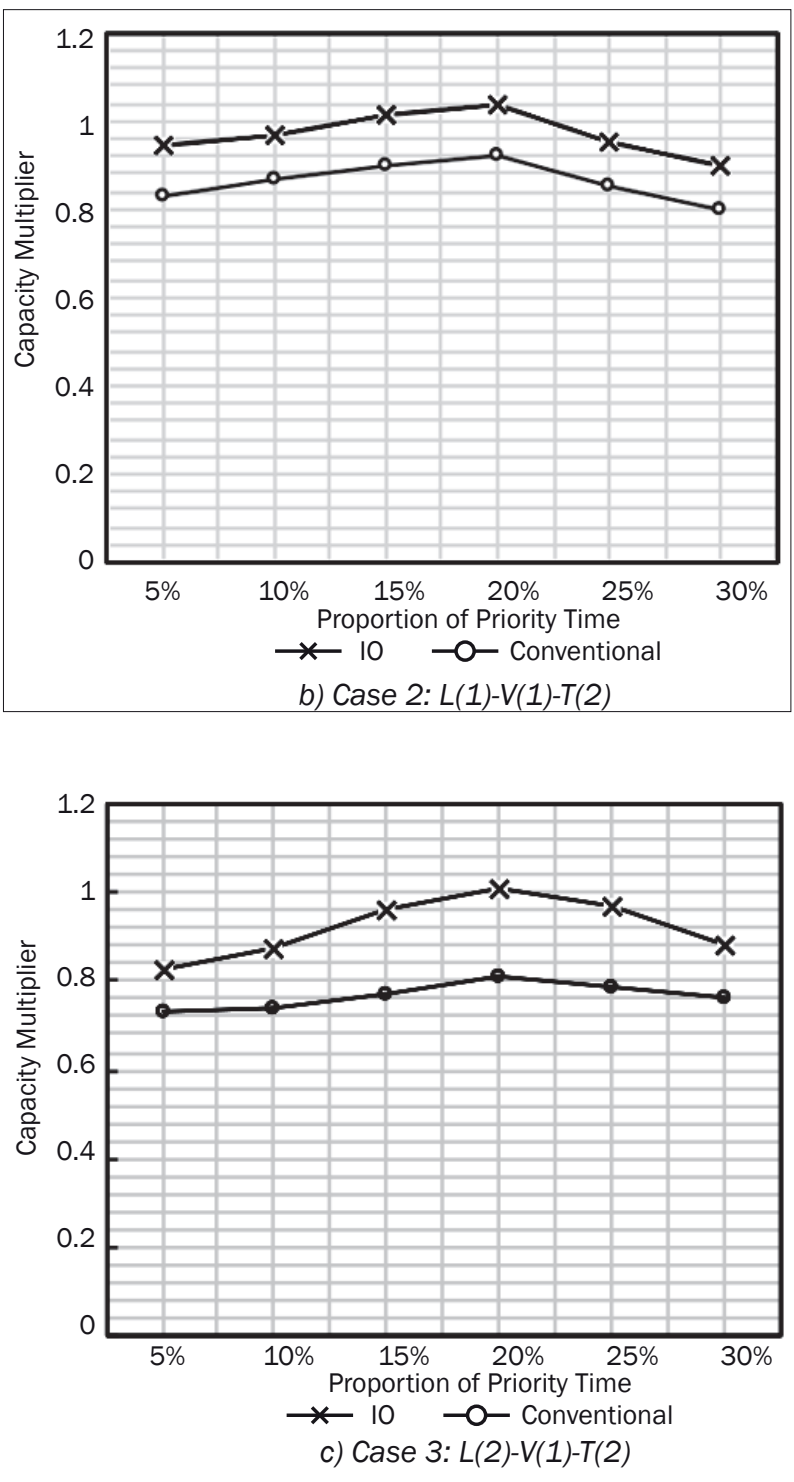

Figure 6 - Numerical analysis results: capacity comparison
Capacity can be increased for vehicles travelling in the same direction as the buses on the basis of the bus signal priority. The redundant time saved by TSP, the variable lanes, and the reduction of through traffic can be reallocated to the left-turn and other phases, so as to increase the capacity of the intersection.

Along with the increase of the left-turn traffic (impaired by TSP) and decrease of the through traffic (benefit from TSP), the utilization of variable lane increases in the proposed 10 method, showing higher capacity multiplier.

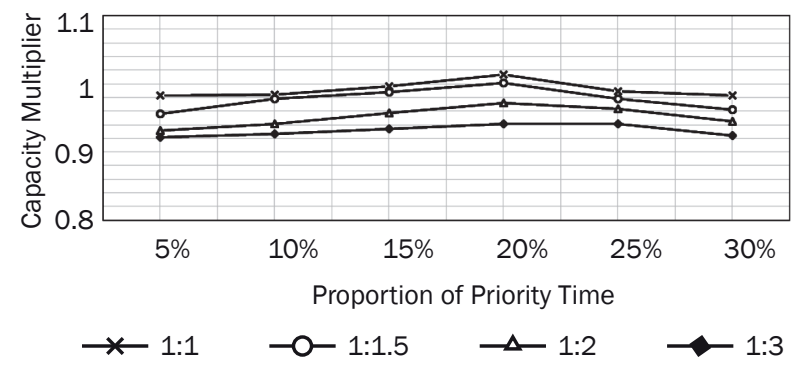

Figure 7 - Numerical analysis results: different left-turn and through flow ratio in Case 1

\subsection{Simulation evaluation}

The geometry layout, traffic volume and signal plan in Case 2 in the numerical analysis are chosen to be the simulation environment. The cycle length is set to be 120 seconds and the variable lane is set to be 50 metres long. Red truncation and 20 seconds are implemented as the priority strategy and priority time, respectively. The frequency of buses is in the range of 2 minutes to 20 minutes. Using the control variable method and keeping the priority strategy and time unchanged, the bus frequency changed in the increments of 2 minutes according to the cycle length. The control variable method can compare the performance of the 10 model to the $\mathrm{OC}$ model under the same bus priority strategy.

Three lines in Figure 8 stand for the proposed integrated optimization (IO) method, conventional priority method and the original control (OC) situation, respectively. As shown in Figure 8, both 10 method and conventional priority method increase the capacity of intersection along with the bus frequency decrease, and the 10 method performed better in maximizing the capacity of intersections. When the bus frequency is lower than 16 minutes/vehicle, the capacity with 10 method could be larger than the OC situation, which means the capacity reduction caused by TSP can be regained by 10 method under certain conditions. Figure 8 also shows the delay of the intersection under different methods, which decreases when bus frequency decreases. The delay of intersection under 10 method is $3.1 \%$ greater than the OC situation when bus frequency is 12 minutes/vehicle, and less than 3\% when the bus frequency decreases. 


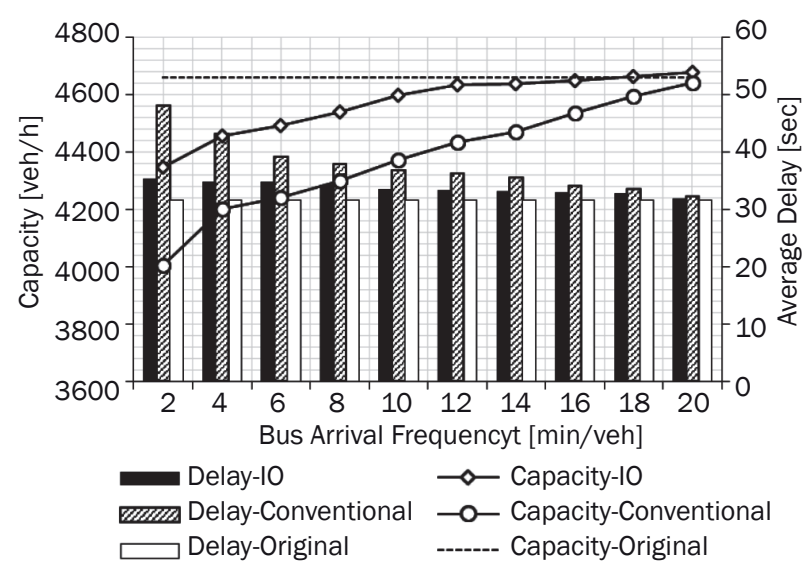

Figure 8 - Simulation results: capacity and delay

Figure 9 shows the performance of the 10 method on capacity increase. The horizontal axis stands for the vehicles input while the vertical axis stands for the vehicles output. Both 10 and conventional priority method performed well when the vehicles input is low, but the 10 model increases more than $8 \%$ of capacity under high saturation degree situation.

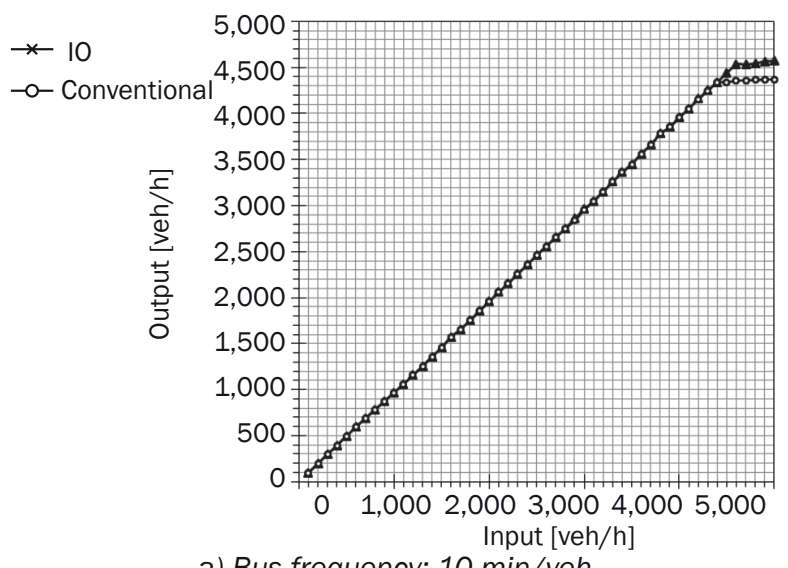

a) Bus frequency: $10 \mathrm{~min} / \mathrm{veh}$

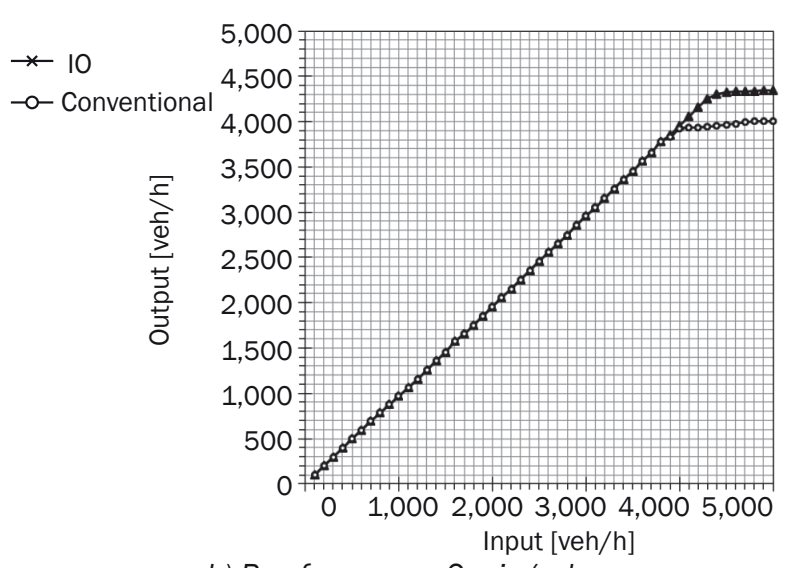

b) Bus frequency: $2 \mathrm{~min} / \mathrm{veh}$

Figure 9 - Simulation test results: throughput and bus frequency impact

\section{CONCLUSION AND FUTURE WORK}

This paper presents an integrated optimization model of lane assignment and traffic signal control for transit progression with DBL. Geometry layout, main-signal timing, pre-signal timing and transit priority are integrated in a unified framework. These problems have been formulated as MINLP and transformed into a series of MILP problem, which can be solved by the standard branch-and-bound technique. Finally, numerical experiments and simulation tests validate the effectiveness of the proposed 10 model. Test results indicate that the proposed 10 method can increase the capacity of intersection in comparison with the conventional bus priority method. A better performance can be achieved when bus frequency is low and left-turn vehicles occupy a relatively large proportion. The intersection delay also receives reduction by the 10 method comparing to the conventional priority method.

In the future work, more extensive numerical experiments, simulation tests can be conducted to assess the performance of the model under various demands and different driving behaviours. Another important work considers the arterial coordination situation in the model to provide integrated optimization in an arterial. The problem of multi-requested priority could be another extension in the future work.

\section{ACKNOWLEDGEMENT}

This research was made possible through the funding by the National Natural Science Foundation of China (Grant No. 61174185 \& Grant No. 51238008).

\section{郝妍熙 滕靖 王吟松 杨晓光}

\section{提升有公交优先措施的交叉口通行能力方法研究}

摘要:

公交专用道和公交信号优先是两种最有效且低 成本的提高公交可靠性的方法。但同时, 这些方法 会降低普通交通的通行能力。本文提出了一种综合 优化模型来提升有公交专用道的信号交叉口的运行 效率。本模型将车道划分, 主信号控制, 预信号控 制及公交优先综合考虑, 形成了一个可以转化为线 型混合整数规划的非线性混合整数规划问题, 本问 题可用分支定界法求解。本文用数理分析和VISSIM 仿真验证了模型的适用性并检验了模型的效率。实 验结果表明本文提出的模型在提高公交运行效率的 同时可以提高交叉口通行能力并减少普通车辆的延 误。

\section{关键词:}

城市道路, 有效的公交信号优先, 公交专用道, 动 态车道划分 


\section{REFERENCES}

[1] Smith W. Westinghouse Airbrake Company, and Institute of Public Administration. Study of Evolutionary Urban Transportation. Volumes I, II, and III; 1968.

[2] Hunter-Zaworski KM, Kloos WC, Danaher AR. Bus priority at traffic signals in Portland: the Powell Boulevard pilot project. Transportation Research Record. 1995;1503:29-33.

[3] Garrow MC, Machemehl RB. Development and evaluation of transit signal priority strategies. Austin: University of Texas; 1997.

[4] Duerr PA. Dynamic right-of-way for transit vehicles: integrated modeling approach for optimizing signal control on mixed traffic arterials. Transportation Research Record: Journal of the Transportation Research Board. 2000;1731(1):31-9.

[5] Furth PG, Muller THJ. Conditional bus priority at signalized intersections: better service with less traffic disruption. Transportation Research Record: Journal of the Transportation Research Board. 2000; 1731(1):23-30.

[6] Janos M, Furth PG. Bus priority with highly interruptible traffic signal control: simulation of San Juan's Avenida Ponce de Leon. Transportation Research Record: Journal of the Transportation Research Board. 2002;1811(1):157-65.

[7] Stevanovic J, Stevanovic A, Martin PT, Bauer T. Stochastic optimization of traffic control and transit priority settings in VISSIM. Transportation Research Part C: Emerging Technologies. 2008;16(3):332-49.

[8] Vlachou K, Collura J, Mermelstein A. Planning and deploying transit signal priority in small and medium-sized cities: Burlington, Vermont, case study. Journal of Public Transportation. 2010;13(3):6.

[9] Wang Y, Ma W, Yin W, Yang X. Implementation and Testing of Cooperative Bus Priority System in Connected Vehicle Environment: Case Study in Taicang City, China. Transportation Research Record: Journal of the Transportation Research Board. 2014;2424:48-57.

[10] Lin W-H. Quantifying delay reduction to buses with signal priority treatment in mixed-mode operation. Transportation Research Record: Journal of the Transportation Research Board. 2002;1811:100-6.

[11] Ngan V, Sayed T, Abdelfatah A. Impacts of various parameters on transit signal priority effectiveness. Journal of Public Transportation. 2004;7(3):71-03.

[12] Ma W, Ni W, Head L, Zhao J. Effective Coordinated Optimization Model for Transit Priority Control Under Arterial Progression. Transportation Research Record: Journal of the Transportation Research Board. 2013;2356(1):71-83.

[13] Hao Y, Wang Y, Yang X. A schedule-based coordinated optimization model for Transit Signal Priority under Connected Vehicle environment. Proceedings of the 17th International Conference on Intelligent Transportation Systems (ITSC); 2014 Oct 8-11; Qingdao, China. p. 2578-2583.

[14] Balke K, Dudek C, Urbanik II T. Development and evaluation of intelligent bus priority concept. Transportation
Research Record: Journal of the Transportation Research Board. 2000;1727:12-9.

[15] Currie G, Lai H. Intermittent and dynamic transit lanes: Melbourne, Australia, experience. Transportation Research Record: Journal of the Transportation Research Board. 2008;2072(1):49-56.

[16] Viegas JM, Roque R, Lu B, Vieira J. Intermittent bus lane system: Demonstration in Lisbon, Portugal. Transportation Research Board 86th Annual Meeting; 2007 Jan 21-25; Washington DC, USA.

[17] Daganzo CF, Laval J, Muñoz JC. Ten strategies for freeway congestion mitigation with advanced technologies. California Partners for Advanced Transit and Highways (PATH); 2002.

[18] Lam WH, Poon AC, Mung GK. Integrated model for lane-use and signal-phase designs. Journal of transportation engineering. 1997;123(2):114-22.

[19] Zhao J, Ma W, Zhang H, Yang X. Two-Step Optimization Model for Dynamic Lane Assignment at Isolated Signalized Intersections. Transportation Research Record: Journal of the Transportation Research Board. 2013;2355:39-48.

[20] Zhao J, Ma W, Zhang H, Yang X. Increasing the capacity of signalized intersections with dynamic use of exit lanes for left-turn traffic. Transportation Research Record: Journal of the Transportation Research Board. 2013;2355:49-59.

[21] Yao D, Su Y, Zhang Y, Li L, Cheng S, Wei Z. Control strategies for transit priority based on queue modeling and surrogate testing. Journal of Intelligent Transportation Systems. 2009;13(3):142-8..

[22] Desnouailles C, Boillon P, Cohen S, Nouvier J. Variable lane assignment: two French projects for minimizing congestion on urban motorways. Pre-Proceedings of the 23rd PIARRC World Road Congress: Choice for Sustainable Development; 2007 Sep 17-21; Paris, France.

[23] Oakes J, Thellmann AM, Kelly IT. Innovative bus priority measures. Proceedings of Seminar J Held at the 22nd PTRC European Transport Forum: Traffic Management and Road Safety. Volume P381; 2014 Sep 12-16; Warwick, England.

[24] Wu J, Hounsell N. Bus Priority Using pre-signals. Transportation Research Part A: Policy and Practice. 1998;32(8):563-83.

[25] Kejun L. Bus priority signal control at isolated intersection. Proceedings of the 2008 International Conference on Intelligent Computation Technology and Automation (ICICTA); 2008 Oct 20-22. p. 234-237.

[26] Xuan Y, Daganzo CF, Cassidy MJ. Increasing the capacity of signalized intersections with separate left turn phases. Transportation research part B: Methodological. 2011;45(5):769-81.

[27] Wong C, Wong S. Lane-based optimization of signal timings for isolated junctions. Transportation Research Part B: Methodological. 2003;37(1):63-84.

[28] Heydecker BG. Sequencing of traffic signals. Institute Of Mathematics \& Its Applications Conference Series 38: Mathematics in Transport Planning and Control; 1992. p. 57-67. 